\title{
3-Dimensional Printed Models May Be a Useful Tool When Planning Revision Anterior Cruciate Ligament Reconstruction
}

\author{
Gene Kitamura, M.D., Marcio Bottene Villa Albers, M.D., Bryson P. Lesniak, M.D., \\ Stephen Joseph Rabuck, M.D., Volker Musahl, M.D., Carol L. Andrews, M.D., \\ Anish Ghodadra, M.D., and Freddie Fu, M.D.
}

\begin{abstract}
Purpose: To determine whether using 3-dimensional (3D)-printed models in addition to computed tomography (CT) scans to evaluate the primary femoral and tibial tunnels before revision anterior cruciate ligament (ACL) reconstruction leads to better agreement with the surgical approach than CT alone. Methods: Fifteen patients who underwent revision ACL reconstruction were retrospectively identified. The mean age was 24.3 years, and $73 \%$ were female. Using only CT images, 3 board-certified orthopaedists and 5 sports medicine orthopaedic fellows evaluated whether the existing tibial and femoral tunnels were acceptable for the revision surgery. Subsequently, 3D-printed models were made available in addition to the CT scan, and the same questions were asked. Results: For the attending orthopaedic physicians, adding the 3D-printed models did not have a significant impact on the tibial or femoral tunnel agreement compared with the surgical approach. With the fellow physicians, however, using the 3D-printed models with tibial tunnel evaluation led to a higher agreement rate $(76 \%)$ compared with CT images alone $(63 \%)(P=.050)$. Furthermore, with the fellow physicians, there was a higher overall agreement when evaluating both the tibial and femoral tunnels with the addition of 3D-printed models $(74 \%)$ compared with CT alone $(65 \%)(P=.049)$. Conclusion: Our hypothesis that using 3D-printed models leads to better agreement with the surgical approach was unsupported based on the response of the board-certified orthopaedists. Based on the fellow response, it stands to reason that 3D-printed models may be a useful tool in understanding spatial orientation when planning for revision ACL surgery. Level of Evidence: IV, retrospective case series.
\end{abstract}

A nterior cruciate ligament (ACL) reconstruction has undergone major advances in the last 30 years, as surgeons better understand the importance of anatomic

From the Department of Radiology (G.K., M.B.V.A., A.G., C.L.A.) and the Department of Orthopedic Surgery, Division of Sports Medicine (B.P.L., S.J.R., V.M., F.F.), University of Pittsburgh Medical Center (UPMC); and the University of Pittsburgh, School of Medicine (G.K., M.B.V.A., A.G., C.L.A.), Pittsburgh, Pennsylvania, U.S.A.

Anish Ghodadra and Freddie Fu are co-last authors.

The authors report the following potential conflicts of interest or sources of funding: Funding for this project was provided by the UPMC Department of Orthopedic Surgery Chairman's fund. A.G. reports nonfinancial support from Axial3D. V.M. reports other from Smith $\theta$ Nephew. Full ICMJE author disclosure forms are available for this article online, as supplementary material.

Received March 31, 2019; accepted June 27, 2019.

Address correspondence to Gene Kitamura, M.D., UPMC Department of Radiology, 200 Lothrop Street, UPMC Montefiore, Room NE 538, Pittsburgh, PA 15213, U.S.A.E-mail: kitamurag@upmc.edu

(C) 2019 by the Arthroscopy Association of North America. Published by Elsevier Inc. This is an open access article under the CC BY-NC-ND license (http://creativecommons.org/licenses/by-nc-nd/4.0/).

2666-061X/19365

https://doi.org/10.1016/j.asmr.2019.06.004 reconstruction. ${ }^{1}$ Despite all the recent advances in the technical aspects of ACL reconstruction, the incidence of revision ACL surgery continues to rise, particularly among young and active patients. ${ }^{2,3}$ Because the most common risk factor for revision has been cited as technical failure, ${ }^{4,5}$ improved preoperative planning and recognition of nonanatomic tunnels from the initial surgery may reduce the risk of graft failure after revision.

Prior studies have shown the benefits of placing the bone tunnels at the anatomic footprints to restore ACL functionality. ${ }^{6,7}$ Femoral tunnel malposition during the index procedure is considered to be the one of the most frequent causes of graft failure in primary ACL reconstructions. ${ }^{3,4,8,9}$ Additional studies have shown that tibial tunnel position is equally important in restoring ACL functionality. ${ }^{10-12}$ Intraoperative fluoroscopy, radiographs, 2-dimensional (2D) and 3D computed tomography (CT), magnetic resonance imaging (MRI), and direct visualization during arthroscopy have all been used to determine the correct tunnel drilling location. . $13-16$ With revision ACL surgery, proper tunnel placement becomes more complex, as 
index tunnels may preclude drilling of subsequent tunnels in an anatomic position. ${ }^{17,18}$ Moreover, tunnel widening may preclude single-stage surgery, requiring a 2 -stage procedure with bone grafting. The higher complexity of revision ACL surgery results in reoperation rates as high as $35 \%$ in patients $<20$ years of age. ${ }^{19,20}$

$3 \mathrm{D}$-printed models have been used in orthopaedic surgery to aid in the management of complex fractures, where spatial orientation plays a crucial role in defining the best surgical strategy. ${ }^{21-25}$ In addition, a cadaveric study has used 3D-printed models to create custom guides to aid in the placement of femoral tunnels during ACL reconstruction. ${ }^{26}$

The purpose of this study was to determine whether using 3D-printed models in addition to CT scans to evaluate the primary femoral and tibial tunnels before revision ACL reconstruction leads to better agreement with the surgical approach than if CT alone is used. Our hypothesis was that incorporating 3D-printed models would lead to better concordance rates.

\section{Materials and Methods}

The study was retrospective in nature and was approved by the local institutional review board with waiver of consent. The inclusion criterion was patients who had revision ACL surgery at our academic institute. The exclusion criteria were incomplete operative records, the presence of multiple femoral and/or tibial tunnels, previous high tibial or distal femur osteotomies, and lack of preoperative CT scan. To preserve blinding, the case selection was performed by a research fellow who was not involved in patient care under the supervision a board-certified orthopaedist; all cases were deidentified before being presented. The final sample consisted of 15 nonconsecutive cases performed by 3 different surgeons ( 5 cases each). The gold standard was the operative approach taken at the time of the ACL revision surgery.

The initial CT scans were ordered for preoperative planning, and 3D reconstructed images were also available. The 3D-printed models were created using CT Digital Imaging and Communications in Medicine (DICOM) files, which were pushed as deidentified cases from our Picture Archive and Communication System to a processing workstation. The files were loaded into Mimics (Materialise NV), a Food and Drug Administration-approved segmentation software. Bone segmentation was performed with a combination of automated thresholding and manual segmentation. The files were then transferred to 3-matic (Materialise $\mathrm{NV}$ ), a stereolithography (STL)-modification software, where hollowed-out 3D models were created. Finally, the STL files were oriented in Preform (Formlabs) and printed on the Form 2 printer, a stereolithography printer from Formlabs. Each model required $\sim 5$ hours to complete printing. The accuracy of each process was validated by a radiologist for every case. The models were not scaled and were printed at actual size. The 3Dprinted models are essentially physical representations of the 3D CT reconstructions.

When the models were done printing, they were postprocessed by washing in isopropyl alcohol and curing in an ultraviolet light oven. At the end, the models were visually inspected for quality assurance (Fig 1). The material cost for each model was $\sim \$ 30$. When accounting for the overhead and labor costs, which included input from radiologists, the total cost for each model was $\sim \$ 300$.

The blinded patient data were presented to 3 boardcertified, sports medicine orthopaedic surgeons (length of career of 7, 10, and 10 years; mean 9 years) and 5 sports orthopaedic fellows (during the final 2 months of their 12-month fellowship) in 2 rounds. The fellows at our institution participate in $\sim 25$ ACL revisions each per year. During the first round, only the CT scans were presented using Horos software V2.4.0 (HorosProject.org). In evaluating the femoral and tibial tunnels, the location, trajectory, and size of the index tunnels were considered. For each case, the participants responded to 2 questions:

1. Can you use the existing tibial tunnel for this patient?

2. Can you use the existing femoral tunnel for this patient?

During the second round, the 3D-printed models were made available in addition to the CT scans; the participants were then presented with the same 2 questions.

The McNemar test was used to compare the responses between using the CT images alone versus incorporating the $3 \mathrm{D}$ models in correctly predicting the surgical approach. Ad hoc power was calculated from the McNemar statistics. One of the attending orthopaedists repeated the second round several months later to evaluate intraobserver reliability (kappa value).

\section{Results}

The 15 selected patients had revision ACL surgery performed in our institution from January 2012 to May 2017. Female patients accounted for $73.3 \%$ (11 of 15) of the cases, and the left side was operated in $66.7 \%$ (10 of 15$)$ of the cases. The mean age was $24.3 \pm 5.0$ years at the time of the CT scan, and the mean time from the primary surgery to the CT sca was $38 \pm 15$ months. Intra-observer reliability of 3D-printed model evaluation showed substantial agreement, with a kappa value of 0.733 . Pre- and postoperative findings for the patients are summarized in Tables 1 and 2.

With the attending orthopaedic physicians, adding the 3D-printed models to CT images did not have a 

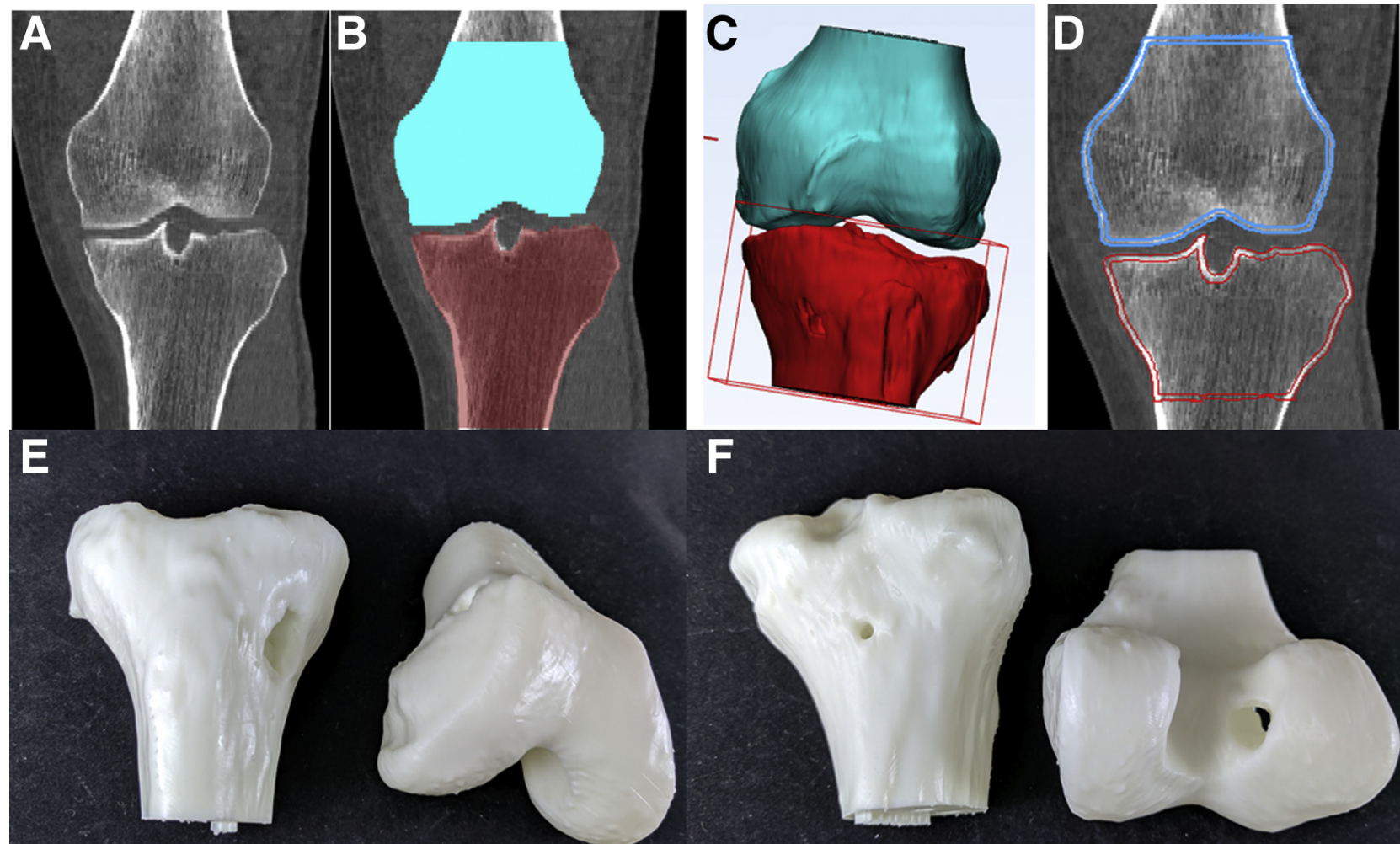

$\mathbf{F}$

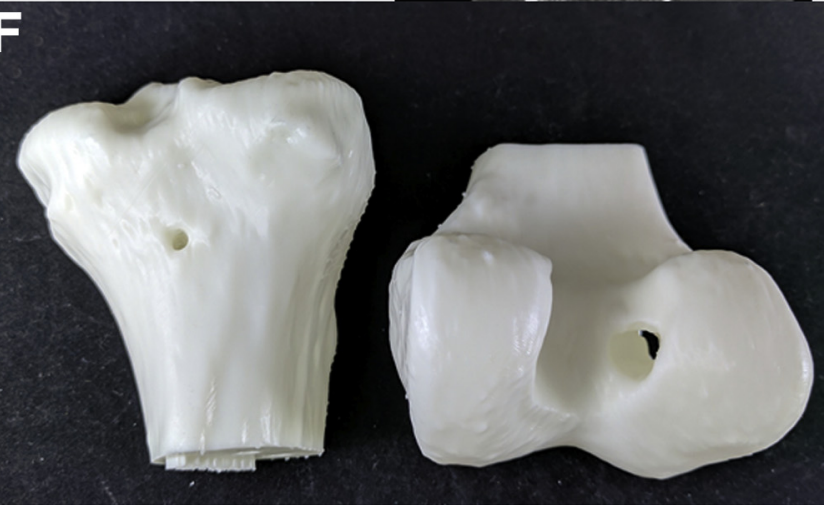

Fig 1. The steps necessary to prepare a CT image for 3D printing. (A) The initial CT image is imported. (B) The right distal femur and proximal tibia are segmented; hardware is manually excluded when present. (C) The segmentation masks are converted to STL files, unnecessary parts are cropped, and the models are hollowed. (D) The final STL files are overlaid on the initial CT to check the model accuracy before printing. (E) An anterior view of the right knee models showing the tibial tunnel. (F) A posterior view of the right knee models showing the femoral tunnel. 3D, 3-dimensional; CT, computed tomography; STL, stereolithography.

significant impact on the tibial or femoral tunnel agreement compared with the surgical approach. With the fellow physicians, however, using the 3D-printed models with tibial tunnel evaluation led to a higher agreement rate with the surgical approach (57 of $75 ; 76 \%$ ) compared with CT images alone $(47$ of $75 ; 63 \%)(P=$
.050). Furthermore, with the fellow physicians, there was a higher overall agreement rate with the surgical approach when evaluating both the tibial and femoral tunnels using the 3D-printed models ( 111 of 150; 74\%) compared with CT alone (98 of 150; 65\%) $(P=.049)$ (Table 3). Study power was only as high as 0.6.

Table 1. Preoperative Findings of the Cases Describing the Graft Used for the Index Procedure and Relevant Information Regarding the Fixation Hardware

\begin{tabular}{|c|c|c|c|}
\hline Patient & Initial Graft & Tibial Hardware & Femoral Hardware \\
\hline 1 & Hamstring autograft & Nonmetal interference screw & Suspensory fixation \\
\hline 2 & Tibialis anterior allograft & Nonmetal interference screw & Suspensory fixation \\
\hline 3 & Hamstring autograft & Nonmetal interference screw & Suspensory fixation \\
\hline 4 & Peroneus longus allograft & Metal cortical screw & Metal tunnel screw \\
\hline 5 & Bone-tendon-bone patellar autograft & Metal interference screw & Suspensory fixation \\
\hline 6 & Bone-tendon-bone patellar autograft & Nonmetal interference screw & Nonmetal interference screw \\
\hline 7 & Hamstring autograft & Nonmetal interference screw & Suspensory fixation \\
\hline 8 & Allograft & Nonmetal interference screw & Suspensory fixation \\
\hline 9 & Allograft & Nonmetal interference screw & Suspensory fixation \\
\hline 10 & Allograft & Nonmetal interference screw & Suspensory fixation \\
\hline 11 & Allograft & Nonmetal interference screw & Suspensory fixation \\
\hline 12 & Bone-tendon-bone patellar autograft & Metal interference screw & Metal interference screw \\
\hline 13 & Hamstring autograft & Nonmetal interference screw & Nonmetal interference screw \\
\hline 14 & Hamstring autograft & Nonmetal interference screw & Suspensory fixation \\
\hline 15 & Hamstring autograft & Nonmetal interference screw & Suspensory fixation \\
\hline
\end{tabular}

Because most of the index procedures were not done at our institute, more specific information about the graft and hardware was not available. 
Table 2. Intraoperative and Postoperative Findings of the Cases Describing Whether the Tunnels Were Reused, Whether Staging of the Procedure Was Required, and Relevant Notes

\begin{tabular}{lccll}
\hline Patient & $\begin{array}{c}\text { Reused Tibial } \\
\text { Tunnel }\end{array}$ & $\begin{array}{c}\text { Reused Femoral } \\
\text { Tunnel }\end{array}$ & Staged & \\
\hline 1 & Yes & No & No & Femoral tunnel bone loss, leading to over-the-top graft placement \\
2 & No & No & Yes & Tunnels too wide with cysts \\
3 & Yes & No & No & Antical femoral tunnel \\
4 & No & No & No & Anterior tibial tunnel and vertical femoral tunnel \\
5 & No & No & No & Posterior tibial tunnel and vertical femoral tunnel \\
6 & No & Yes & No & \\
7 & Yes & No & No & Posterior tibial tunnel and vertical femoral tunnel \\
8 & No & No & Yes & Tunnels too wide \\
9 & No & Yes & No & \\
10 & Yes & Yes & No & \\
11 & Yes & No & No & Nonanatomic tunnels \\
12 & No & No & No & Posterior tibial tunnel and anterior femoral tunnel \\
13 & No & No & No & Anterior and horizontal femoral tunnel \\
14 & Yes & No & No & Vertical femoral tunnel \\
15 & Yes & &
\end{tabular}

*Operative note for patient 12 was sparse, noting only that the index tunnels were not at the anatomic footprints.

A couple of cases showed marked fellow response changes. Using 3D-printed models changed a nonconcordant response to a concordant one for all 5 fellows when evaluating the femoral tunnel for patient 4 , which was too vertical, and the tibial tunnel for patient 6 , which was too posterior. In addition, 4 of the 5 fellows changed their nonconcordant response to a concordant one when using 3D-printed models to evaluate the tibial tunnel for patient 10, which reused the index tunnel. Interestingly, using a 3D-printed model did not show a compelling response change for the staged, bone-grafted cases with widened tunnels (patients 2 and 9).

\section{Discussion}

Based on the response of the board-certified orthopaedists, our hypothesis that using 3D-printed models leads to better agreement with surgical approached was not supported. At the same time, our study suggests that fellow physicians, and by extension resident physicians in training, may benefit from using 3D-printed models as an adjunct learning tool with revision ACL reconstruction. The use of 3D-printed models had the most impact when evaluating the tibial tunnel, but there also was evidence showing increased overall agreement with operative findings when using both the 3D printed tibial and femoral models with the fellows. The lack of change seen in the board-certified, attending orthopaedic physicians is attributed to their expertise, experience, and familiarity with the procedure compared with the fellow physicians. Although speculative, 3D-printed models may be useful to early career sports physicians and other physicians who may be board certified but not as familiar with these types of cases.

Several cases led to a high number of nonconcordant responses changing to concordant ones for the fellows after the 3D-printed model was added to the workup (femoral tunnel for patient 4 and tibial tunnel for patients 6 and 10). This finding suggests that 3D-printed models may have a higher impact in certain cases where the spatial orientation is much better understood with actual physical models rather than from just images. At the same time, 3D-printed models may not offer much value with cases showing obviously widened tunnels requiring a staged procedure, which are often discernable on imaging.

The practical implication of this study is to consider using 3D-printed models as an adjunct tool when planning for revision ACL reconstruction cases. Although the total cost of the whole model was $\sim \$ 300$, cost reduction may be possible once these cases are printed more routinely, as less input will be needed from higher-level operators such as radiologists, and may reduce costs to $<\$ 100$ a case.

Table 3. Number of Cases in Agreement With the Surgical Approach for Tibial and Femoral Tunnels Using CT Alone Versus Added 3D-Printed Model

\begin{tabular}{|c|c|c|c|}
\hline & CT Alone & $\begin{array}{c}\text { CT Plus } \\
\text { 3D-Printed } \\
\text { Model }\end{array}$ & $\begin{array}{c}P \text { Value, 1-Tail } \\
\text { McNemar's Test }\end{array}$ \\
\hline \multicolumn{4}{|l|}{ Tibial tunnel agreement } \\
\hline All physicians & 79 & 88 & 0.111 \\
\hline Only attending physicians & 32 & 31 & 0.500 \\
\hline Only fellow physicians & 47 & 57 & $0.050^{*}$ \\
\hline \multicolumn{4}{|l|}{ Femoral tunnel agreement } \\
\hline All physicians & 85 & 85 & 0.428 \\
\hline Only attending physicians & 34 & 31 & 0.225 \\
\hline Only fellow physicians & 51 & 54 & 0.338 \\
\hline \multicolumn{4}{|l|}{ Overall agreement } \\
\hline All physicians & 164 & 173 & 0.175 \\
\hline Only attending physicians & 66 & 62 & 0.251 \\
\hline Only fellow physicians & 98 & 111 & $0.049^{*}$ \\
\hline
\end{tabular}




\section{Limitations}

The main limitation of this study is its retrospective nature; it is difficult to truly assess whether a different surgical approach would have been performed had the 3D-printed model been available prospectively. The study was also underpowered (power of 0.6) because of a small sample size, implying a relatively high chance of type 2 error. It is possible that with a higher number of cases, we would have seen a more dramatic response from incorporating the 3D-printed models.

\section{Conclusions}

Our hypothesis that using 3D-printed models leads to better agreement with the surgical approach was unsupported based on the response of the board-certified orthopaedists. However, based on the fellow response, it stands to reason that 3D-printed models may be a useful tool in understanding spatial orientation when planning for revision ACL surgery.

\section{References}

1. Fu FH, van Eck CF, Tashman S, Irrgang JJ, Moreland MS. Anatomic anterior cruciate ligament reconstruction: a changing paradigm. Knee Surg Sports Traumatol Arthrosc 2015;23:640-648

2. Kaeding CC, Aros B, Pedroza A, et al. Allograft versus autograft anterior cruciate ligament reconstruction: predictors of failure from a MOON prospective longitudinal cohort. Sports Health 201 1;3:73-81.

3. Achtnich A, Ranuccio F, Willinger L, et al. High incidence of partially anatomic tunnel placement in primary singlebundle ACL reconstruction. Knee Surg Sports Traumatol Arthrosc April 2017:1-6.

4. Burnham JM, Herbst E, Pauyo T, et al. Technical considerations in revision anterior cruciate ligament (ACL) reconstruction for operative techniques in orthopaedics. Oper Tech Orthop 2017;27:63-69.

5. Chen JL, Allen CR, Stephens TE, et al. Differences in mechanisms of failure, intraoperative findings, and surgical characteristics between single- and multiple-revision ACL reconstructions: a MARS cohort study. Am J Sports Med 2013;41:1571-1578.

6. Ho JY, Gardiner A, Shah V, Steiner ME. Equal kinematics between central anatomic single-bundle and doublebundle anterior cruciate ligament reconstructions. Arthroscopy 2009;25:464-472.

7. Kopf S, Forsythe B, Wong AK, Tashman S, Irrgang JJ, Fu FH. Transtibial ACL reconstruction technique fails to position drill tunnels anatomically in vivo 3D CT study. Knee Surg Sports Traumatol Arthrosc 2012;20:2200-2207.

8. Matava MJ, Arciero RA, Baumgarten KM, et al. Multirater agreement of the causes of anterior cruciate ligament reconstruction failure: a radiographic and video analysis of the MARS cohort. Am J Sports Med 2015;43: 310-319.
9. Morgan JA, Dahm D, Levy B, Stuart MJ. Femoral tunnel malposition in ACL revision reconstruction. J Knee Surg 2012;25:361-368.

10. Bedi A, Altchek DW. The "footprint" anterior cruciate ligament technique: an anatomic approach to anterior cruciate ligament reconstruction. Arthroscopy 2009;25: 1128-1138.

11. Bedi A, Musahl V, Steuber V, et al. Transtibial versus anteromedial portal reaming in anterior cruciate ligament reconstruction: an anatomic and biomechanical evaluation of surgical technique. Arthroscopy 2011;27: 380-390.

12. Brophy RH, Pearle AD. Single-bundle anterior cruciate ligament reconstruction: a comparison of conventional, central, and horizontal single-bundle virtual graft positions. Am J Sports Med 2009;37:1317-1323.

13. Inderhaug E, Larsen A, Waaler PA, Strand T, Harlem T, Solheim E. The effect of intraoperative fluoroscopy on the accuracy of femoral tunnel placement in single-bundle anatomic ACL reconstruction. Knee Surg Sports Traumatol Arthrosc 2017;25:1211-1218.

14. Kosy JD, Mandalia VI. Plain radiographs can be used for routine assessment of ACL reconstruction tunnel position with three-dimensional imaging reserved for research and revision surgery. Knee Surg Sports Traumatol Arthrose 2018;26:534-549.

15. Vermesan D, Inchingolo F, Patrascu JM, et al. Anterior cruciate ligament reconstruction and determination of tunnel size and graft obliquity. Eur Rev Med Pharmacol Sci 2015;19:357-364.

16. Lee D-H, Kim H-J, Ahn H-S, Bin S-I. Comparison of femur tunnel aperture location in patients undergoing transtibial and anatomical single-bundle anterior cruciate ligament reconstruction. Knee Surg Sports Traumatol Arthrosc 2016;24:3713-3721.

17. Tscholl PM, Biedert RM, Gal I. Radiological evaluation for conflict of the femoral tunnel entrance area prior to anterior cruciate ligament revision surgery. Int Orthop 2014;38:607-615.

18. Group, Mars. Factors influencing graft choice in revision anterior cruciate ligament reconstruction in the MARS group. J Knee Surg 2016;29:458-463.

19. Wright RW, Dunn WR, Amendola A, et al. Anterior cruciate ligament revision reconstruction: two-year results from the MOON cohort. J Knee Surg 2007;20: 308-311.

20. Ding DY, Zhang AL, Allen CR, et al. Subsequent surgery after revision anterior cruciate ligament reconstruction: rates and risk factors from a multicenter cohort. Am J Sports Med 2017;45:2068-2076.

21. Zheng W, Tao Z, Lou Y, et al. Comparison of the conventional surgery and the surgery assisted by $3 \mathrm{D}$ printing technology in the treatment of calcaneal fractures. J Invest Surg 2017;19:1-11.

22. Lou Y, Cai L, Wang C, et al. Comparison of traditional surgery and surgery assisted by three dimensional printing technology in the treatment of tibial plateau fractures. Int Orthop 2017;41:1875-1880. 
23. Zeng C, Xiao J, Wu Z, Huang W. Evaluation of threedimensional printing for internal fixation of unstable pelvic fracture from minimal invasive para-rectus abdominis approach: a preliminary report. Int J Clin Exp Med 2015;8:13039-13044.

24. Flecher X, Migaud H. From radiographs to 3D printing: how can new surgical planning technologies contribute to hip surgery? Orthop Traumatol Surg Res 2017;103:323-324.
25. Zerr J, Chatzinoff Y, Chopra R, Estrera K, Chhabra A. Three-dimensional printing for preoperative planning of total hip arthroplasty revision: case report. Skeletal Radiol 2016;45:1431-1435.

26. Ni J, Li D, Mao M, et al. A method of accurate bone tunnel placement for anterior cruciate ligament reconstruction based on 3-dimensional printing technology: a cadaveric study. Arthroscopy 2018;34:546-556. 